\title{
Impulsando la desigualdad «de mercado»: el vínculo elite-Estado en Chile en el siglo $\mathrm{XX}^{*}$
}

\author{
Javier E. Rodríguez Weber**
}

UNIVERSIDAD DE LA REPÚBLICA - URUGUAY

\section{RESUMEN}

El presente texto pretende ser un aporte al esfuerzo de reorientar el análisis de la distribución del ingreso desde un abordaje centrado en los mercados hacia otro que enfatice el rol de la economía política de la desigualdad. Apelando a nueva evidencia sobre la evolución histórica de la desigualdad de ingreso en Chile, en el mismo se comparan los dos episodios de incremento en la desigualdad en los ingresos de mercado (market income) que se produjeron en ese país durante el siglo XX (1903/1913 y 1973/1986). Se muestra que, si bien los factores de mercado jugaron un papel, el rápido y radical incremento de la desigualdad que se produjo en ambos períodos fue consecuencia de la forma histórica en que se resolvió el conflicto distributivo entre distintos sectores de la sociedad. Fue determinante, en particular, la capacidad de la elite de influir en el gobierno del Estado, de modo que éste volcara todo su peso normativo y coercitivo en su favor.

Palabras clave: desigualdad, Chile, ingresos de mercado, instituciones, economía política.

\section{Abstract}

The aim of the paper is to make a contribution to the "political economy turn" that can be seen in the recent literature on income inequality. Using new evidence on market income distribution in Chile since the mid $19^{\text {th }}$ century, the paper analyzes two $20^{\text {th }}$ century episodes of increasing income inequality (1903/1913 and 1973/1986). The paper shows that, although market forces played a role, the rapid increases in income inequality in those years were the result of distributive conflicts between social actors and of the way the state intervened in those conflicts. Furthermore, the power of members of the elite, which allowed them to influence state action in their own favor, was decisive. Keywords: inequality, Chile, market income, institutions, political economy

\footnotetext{
**javier.rodriguez@cienciassociales.edu.uy
} 


\section{INTRODUCCIÓN}

a desigualdad vuelve a estar en el centro de la agenda académica y política. A fines de los ańos noventa, Atkinson (1997) señaló que los economistas estaban retomando su interés en la misma; pero lo que la trajo de vuelta fue -en gran medida- la crisis que luego de 2008 afectó al mundo desarrollado. Esta no sólo empujó a millones de personas a la pobreza -en países que creían haberse librado de ella para siempre-, sino que la pauperización a la que dio lugar ocurrió en medio de la opulencia. Quizá ello explique -en parte- por qué la publicación en 2014 de la edición en inglés de un largo libro académico sobre la historia y el futuro de la desigualdad se transformó en tema de debate público.

Una consecuencia de este renacer de la preocupación por la desigualdad ha sido el reconocimiento de que se trata de un fenómeno tanto político como económico. No es que ello sea ninguna novedad, pero no deberíamos subestimar la importancia de volver a aprender aquellas verdades que habíamos olvidado. Y ocurre que es justamente cuando su carácter político se hace evidente que la desigualdad se ubica en el centro de la agenda. Así ha ocurrido tanto en la segunda mitad del siglo XIX, en los años veinte, o en los inicios del siglo XXI. Por otra parte, este reconocimiento de lo político no debe interpretarse como un desconocimiento de los factores que suelen considerarse propiamente económicos -simplificando: aquellos relacionados a la acción de los mercados. Pero sí que los mercados- nunca actúan en un vacío institucional, sino que -por el contrario- el intercambio está determinado en parte por las relaciones de poder, siempre asimétricas, que existen entre los agentes. Una realidad que suele dar lugar a un conflicto distributivo que en ocasiones se torna violento.

Esta reivindicación de lo político ha venido a desafiar lo que era hasta hace poco la aproximación estándar al problema, es decir, la idea de que los cambios en la distribución del ingreso se explican fundamentalmente como la consecuencia de cambios en la oferta y demanda de factores (Atkinson and Bourguignon 2007) -razón por la cual denominaremos "mercadocéntricos» a este tipo de abordajes-. 
En tanto que la información proveniente de encuestas de hogares muestra a la desigualdad salarial como el principal determinante de la desigualdad global ${ }^{1}$, muchos estudios se concentran en los efectos distributivos de los cambios en el mercado de trabajo. En este sentido, se ha sostenido que las tendencias distributivas son el resultado de una carrera que se produce entre el cambio tecnológico -que incrementa la demanda por calificación-y la expansión educativa -que incide sobre su oferta- (Tinbergen 1975; Goldin and Katz 2009; Kahhat 2010). En forma similar, se sostiene que los procesos de globalización inciden en los patrones de oferta y demanda de factores, incidiendo en su retribución relativa (Beyer et al. 1999). Sin embargo, estos abordajes tienden a desconocer que tanto los procesos de cambio técnico, como de globalización, han estado históricamente asociados a factores sociales e institucionales sin los cuales no se pueden comprender adecuadamente las tendencias distributivas observadas (Robinson 2003; Bértola, Castelnovo et al. 2010).

Estas dificultades han ambientado un "giro institucionalista» en las ciencias sociales que ha alcanzado también a los estudios sobre la desigualdad. Como consecuencia, en los últimos años se han producido un cúmulo de trabajos sobre el rol de las instituciones -y más en general de las políticas-, en la distribución del ingreso (Robinson 2010). Se retoma así una línea de investigación que, si bien de larga tradición en las ciencias sociales, había sido dejada de lado por la economía neoclásica, para la cual el análisis de las fuerzas de mercado resultaba suficiente para explicar lo que ocurriera con la desigualdad. Aunque muchas críticas puedan hacerse a los autores del giro institucionalista, éstos han tenido la virtud de reintroducir el problema del poder en la economía convencional, acercando ésta a perspectivas heterodoxas como el marxismo (Acemoglu, Johnson et al. 2005; Bértola 2011; Acemoglu and Robinson 2012). Así, al hacer de las pugnas distributivas y las relaciones de poder entre los actores sociales los determinantes principales de las instituciones -y de estas la "causa fundamental" del crecimiento y la distribución-, han adscrito a la idea clásica que hace del conflicto social el «motor 
de la historia». De esta forma, ha ido ganando terreno la idea de que, para comprender las dinámicas históricas de la desigualdad, se hace necesario estudiar su economía política (Atkinson 2015; Piketty 2014, 2015).

El presente texto pretende ser un aporte al esfuerzo de reorientar el análisis de la distribución del ingreso desde un abordaje centrado en los mercados hacia otro que enfatice el rol de la economía política de la desigualdad. Apelando a nueva evidencia sobre la evolución histórica de la desigualdad de ingreso en Chile, en el mismo se comparan los dos episodios de deterioro de la distribución que se produjeron en ese país durante el siglo XX: a principios de siglo uno, setenta años después el otro. Se demostrará que, si bien los factores de mercado jugaron un papel, el rápido y radical incremento de la desigualdad que se produjo en ambos períodos fue consecuencia de la forma histórica en que se resolvió el conflicto distributivo entre distintos sectores de la sociedad. Fue determinante, en particular, la capacidad de la elite de influir en el gobierno del Estado, de modo que éste volcara todo su peso normativo y coercitivo en su favor. Ello constituyó el mecanismo clave que propició el cambio de nivel que se produjo en la desigualdad en ambos períodos. De este modo, tanto en el Chile anterior a la Primera Guerra Mundial, como en el de los años setenta y ochenta del siglo XX, la desigualdad se incrementó porque existieron una serie de decisiones de política que no sólo hacían del deterioro de la distribución un resultado probable, sino previsible.

\section{DeFINICIÓN DEL PROBLEMA: LA ECONOMÍA POLÍTICA DE LA DES-} IGUALDAD

La acción política y el marco institucional pueden incidir en la distribución del ingreso de dos maneras. Existe, por una parte, la redistribución que se realiza mediante el sistema impositivo, el gasto público o las transferencias. Se le denomina en ese caso «distribución secundaria» y es el tipo de redistribución más estudiado. La mayor parte de los trabajos que analizan la distribución secundaria comparten con los enfoques «mercadocéntricos» el supuesto de que 
del mercado se deriva una "distribución primaria» determinada por los mecanismos neoclásicos habituales -la retribución a cada factor es equivalente a su aportación marginal al proceso productivo-. Se supone que es sobre esta distribución de mercado que opera la acción política cuyos efectos interesa estudiar.

Sin embargo, ningún mercado opera en un vacío institucional, por lo que no existe una distribución primaria para la cual sólo la oferta y demanda de factores sea lo relevante. De ahí que exista un segundo conjunto de mecanismos por los cuales el marco institucional y la acción política de los diversos agentes -sea el Estado, grupos de interés o individuos-, incide en la distribución del ingreso. Estos operan "dentro» de la distribución primaria o de mercado. En otras palabras, el rol de las instituciones y las políticas en la desigualdad no se limita a su incidencia en el ingreso disponible -luego de impuestos y transferencias- sino que incide también en la distribución primaria o «de mercado». Y es justamente la economía política de la distribución «de mercado» lo que nos proponemos abordar. Este se estudia en las dos ocasiones en la historia de Chile en el siglo XX en que se observó un importante aumento de la desigualdad.

¿Por qué no existe una distribución del ingreso primaria derivada exclusivamente de la oferta y demanda de factores? En la generación y distribución del «ingreso de mercado» hay mucho más que oferta y demanda de factores. Los agentes que se encuentran en el mercado -por ejemplo capitalistas y trabajadores, terratenientes y arrendadores, o prestamistas y deudores- no sólo son compradores y vendedores. Existen entre ellos, además de la relación de intercambio, relaciones de poder y subordinación que se expresan, entre otras cosas, en su capacidad de incidir en el precio de los factores, diferenciándolo así del que se fijaría en un contexto de competencia perfecta -un concepto en exceso simplificador que deja de lado factores clave para el resultado distributivo-. Junto a estas relaciones de poder entre los agentes, existen también las instituciones y políticas que, al regular las relaciones de intercambio, influyen en la retribución a los factores. Las reglas que rigen los derechos de propiedad sobre los activos, las instituciones del mercado de trabajo -como la existencia o no de negociación colectiva, 
las normas que regulan la actividad sindical o las que inciden en la fijación del salario, como los salarios mínimos o los mecanismos de reajuste- son parte constitutiva de los ingresos «de mercado»e inciden su distribución. También el Estado contribuye a moldear la distribución primaria, tanto por su incidencia en las instituciones formales que regulan los mercados de factores, como por su participación directa en el conflicto distributivo entre los actores económico-sociales, sea reprimiendo una huelga o presionando a los empresarios para que concedan un aumento salarial mayor al que desearían. Dicha incidencia interactúa con los mecanismos de mercado en un sentido que puede reforzar, aminorar, o incluso revertir las tendencias distributivas que, en ausencia de factores institucionales, se derivarían de la evolución de la oferta y la demanda.

Los dos episodios históricos que analizaremos en este trabajo se caracterizaron por un fuerte incremento de la desigualdad de mercado - toda la evidencia que presentaremos refiere a distribución antes de impuestos y transferencias- pero dicho incremento no se debió, ni exclusiva ni fundamentalmente, a cambios en la oferta y demanda de factores, sino al resultado del conflicto distributivo entre distintos sectores de la sociedad. Un conflicto que asumió en ambas ocasiones un carácter violento y que tuvo en el Estado -que intervino en los dos episodios a favor de la elite- un actor fundamental.

Sin embargo, de lo anterior no se sigue necesariamente la ausencia o irrelevancia de factores de mercado como parte de la explicación de lo ocurrido con la desigualdad en los dos períodos analizados. Lo que sí sostenemos es que una explicación basada en ellos -como la que caracteriza a los trabajos inspirados en el enfoque «mercadocéntrico»- resulta insuficiente. De este modo, nuestro objetivo no consiste brindar una explicación completa de lo ocurrido con la desigualdad en ambos períodos, sino demostrar la incidencia de factores que han sido subestimados por la literatura neoclásica sobre la distribución del ingreso ${ }^{2}$, pero cuya relevancia viene siendo señalada con creciente énfasis por la literatura más reciente sobre la desigualdad (Atkinson 2015; Piketty 2014, 2015). 


\section{DOS EPISODIOS DE INCREMENTO DE LA DESIGUALDAD EN EL SIGLO XX Los Datos}

Las estadísticas de distribución del ingreso que se presentan en este estudio forman parte de la estimación de indicadores de ingreso y desigualdad para un período más amplio y resulta de la combinación de distintas metodologías (Gráfico 1). La desigualdad de ingreso entre 1974 y 1989 se ha estimado a partir de una fuente estándar, la Encuesta de Ocupación, Desocupación y Percepción de Ingresos y Gastos del Gran Santiago, por lo que no requiere mayores explicaciones, salvo destacar que no se trata de información recabada a nivel nacional, la que sólo está disponible para el período posterior. Sí es necesario presentar, aunque sea en forma somera, la metodología seguida para la estimación del período 1903-1918.

La estimación de los índices de ingreso y desigualdad para el período 1860-1970 se deriva de la construcción de dos tablas sociales, una metodología que ha sido utilizada en un amplio número de casos (Lindert and Williamson 1982; Londoño 1995; Bértola 2005; Milanovic 2010; Lindert and Williamson 2014). Una tabla social supone elaborar una matriz compuesta por el número de personas que cuentan con ingresos y el monto de éstos, agrupadas en distintas categorías sociales y/o ocupacionales. Una peculiaridad de las tablas sociales estimadas es que son dinámicas, esto es que cubren un período de tiempo en lugar de un año específico. Ello constituye el elemento dinámico, mientras la persistencia de las mismas categorías brinda coherencia a la construcción y permite las comparaciones intertemporales ${ }^{3}$.

La tabla social cuya estimación se utiliza aquí para analizar el primer episodio de incremento de la desigualdad en el siglo XX cubre el período 1860-1930 y se divide en 49 categorías de perceptores de ingreso: 9 en la agricultura -7 categorías de terratenientes y dos de trabajadores-, 3 en la minería, 10 en la industria, 2 en el transporte, 20 en el Estado, y 2 de otros -profesionales y sirvientes-. La principal fuente utilizada es la información de personas "con profesión" que recogen los Censos de Población. Respecto a otras fuentes, siguen en importancia los Anuarios Es- 
tadísticos y las estadísticas económicas históricas. Finalmente, se ha recurrido a un conjunto de información dispersa en bibliografía secundaria ${ }^{4}$.

\section{LOS RESULTADOS}

El análisis de largo plazo permite realizar comparaciones entre distintos períodos, echando luz sobre factores de larga duración que, como el vínculo Estado-elites, suele ser considerado como uno de los anclajes de la elevada desigualdad que caracteriza a Latinoamérica. El Gráfico 1 presenta la serie completa de desigualdad de ingreso en Chile entre 1850 y 2009 . El mismo permite apreciar que, en lo que refiere al siglo XX, Chile vivió dos períodos de deterioro de la distribución del ingreso: entre 1903 y 1918 y entre 1974 y 1989 -aproximadamente-. Ambos son presentados en el Gráfico 2.

Aunque las diferentes metodologías utilizadas para la estimación de los dos períodos -tablas sociales en 1903-1918 y encuestas de hogares para 1974-1989- obliga a ser cauteloso a la hora de comparar los resultados, el Gráfico 2 permite apreciar que ambos episodios de incremento de la desigualdad de ingreso tienen al menos dos características comunes. En los dos casos el deterioro fue importante y rápido, al punto que -medida por el índice de Gini- la desigualdad empeoró en el entorno de 15 puntos porcentuales en algo más de una década. Vistos en perspectiva de más largo plazo, ambos episodios supusieron -además- una rápida reversión de sendas tendencias de mejora en la distribución que se habían constatado en las décadas anteriores (Gráfico 1).

Desde el punto de vista institucional, ambos períodos tienen en común el tratarse de regímenes no democráticos. El régimen político imperante durante el primero es conocido como la República Oligárquica. Durante la misma, se ha dicho, "el predomino de la aristocracia o clase rectora se hace absoluto [al asumir] la totalidad del poder político", algo que ocurre a partir de la derrota de Balmaceda en 1891 (Vial Correa 2010, 992; Reinsch 1909). El segundo período, signado por la dictadura encabezada por el General Augusto Pinochet -quien asumiera el mando del Ejecutivo tras el derroca- 
miento del presidente Salvador Allende- supuso la instauración de un régimen de terror bajo cuyo amparo se llevaron adelante medidas tendientes no sólo a la destrucción de toda oposición política y social -especialmente partidos de izquierda y sindicatos- sino a la transformación radical del ordenamiento económico e institucional (Gárate Chateau 2012).

En la próxima sección nos abocamos a realizar un análisis comparado de los mecanismos de economía política que favorecieron en ambos períodos el deterioro de la distribución del ingreso «de mercado».

Gráfico 1: Desigualdad personal del ingreso «de mercado» en Chile en el largo plazo, 1850-2009. Índice de Gini

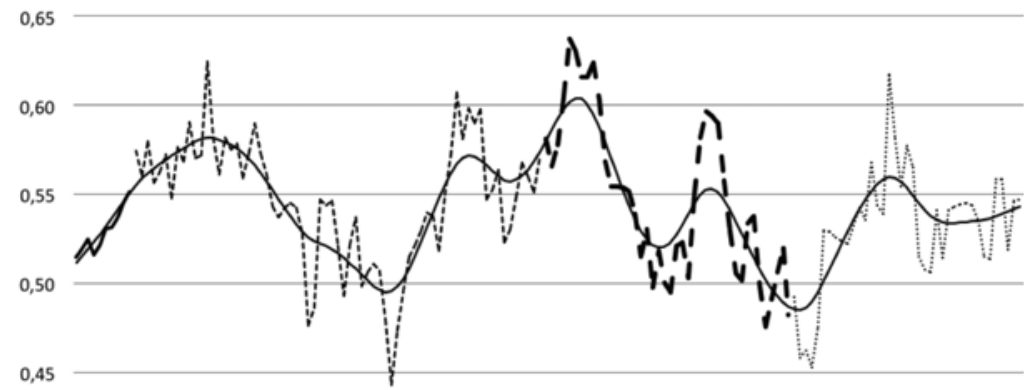

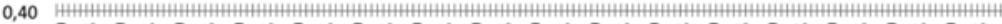

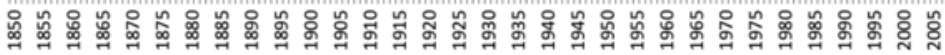

\section{- -(1850-1860) Estimado según Ratio de Extracción \\ - (1860-1929) Estimado partir de Tabla Social 1860-1930 \\ - - (1929-1970) Estimado a partir de Tabla social 1929-1970 \\ (1970-2010) Estimado a partir de la Encuesta de Ocupación en Gran Santiago \\ - Tendencia Hodrick-Prescott (parámetro de suavización 100)}

Fuentes: 1850-1970, estimación propia. 1971-2009, Calculado entre personas que perciben ingresos a partir de la "Encuesta de Ocupación, Desocupación y Percepción de Ingresos y Gastos del Gran Santiago", elaborada por la Universidad de Chile. El cambio en el trazo de la serie original señala las diferentes metodologías y fuentes utilizadas. De lo anterior se deriva la necesidad de ser cauteloso a la hora de extraer conclusiones relativas a los niveles de la desigualdad para períodos estimados con metodologías diferentes; las tendencias resultan más confiables. Para un análisis de la sensibilidad de los resultados ante cambios metodológicos ver Rodríguez Weber (2014: Capítulo 3) 
Gráfico 2: Dos episodios de deterioro de la distribución del ingreso en Chile en el siglo XX: 1903-1918 y 1974-1989. Índice de Gini

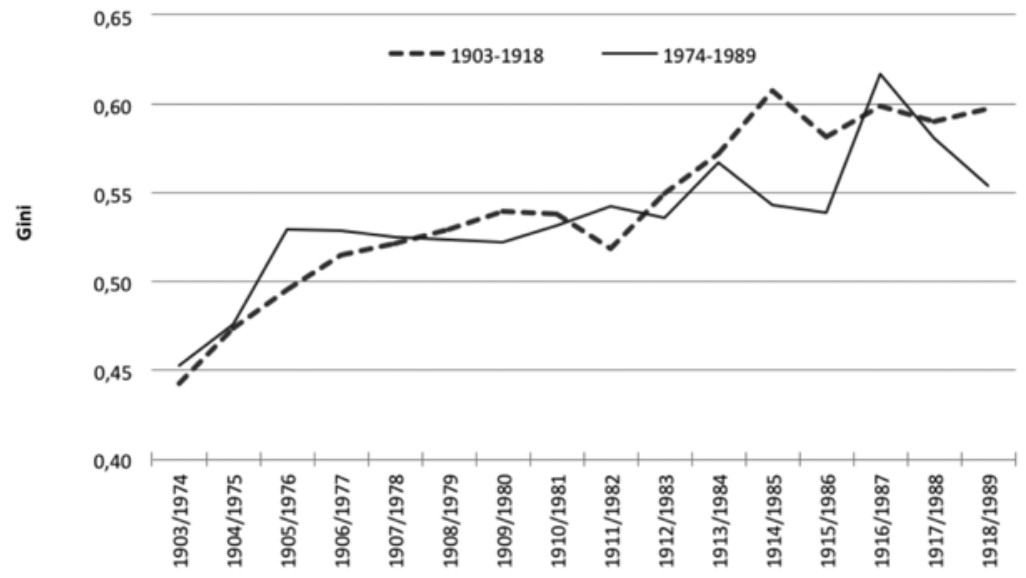

Fuente: Elaboración propia

El víNCULO ELITES-ESTADO Y LOS MECANISMOS ECONÓMICO-POLÍTICOS DE DISTRIBUCIÓN REGRESIVA

El vínculo privilegiado entre la elite y el Estado constituye un factor relevante en la explicación del deterioro de la desigualdad que se observa en ambos períodos. Dicho vínculo contribuyó a provocar el incremento de la desigualdad mediante dos tipos de mecanismos que, si bien comunes a ambos, adquirieron características específicas en cada uno de ellos. Por un lado, estuvo la incidencia en el mercado de trabajo. Ésta supuso que quienes gobernaban el Estado intervinieran en el conflicto distributivo en un sentido favorable a la elite y contrario a los asalariados, fuera reprimiendo a los trabajadores, prohibiendo la actividad sindical y/o imponiendo un régimen laboral tendiente a garantizar la sumisión del trabajador respecto del patrón. En segundo lugar, estuvo la privatización de recursos y servicios públicos en contextos opacos, lo que propició -en ambos períodos- la concentración de la propiedad y la ampliación de la esfera de negocios de la elite. 
VOLCANDO El PESO COERCITIVO DEL EsTAdo A FAVOR DE LA ELITE Entre 1903 y 1913, el ingreso real medio por perceptor creció al $1,9 \%$ anual, mientras el del 1\% superior lo hizo al 8\%. En el otro extremo, los trabajadores no calificados perdieron ingreso real a una tasa de $-1,5 \%$ anual. Como resultado, el ingreso real total se incrementó en un 33\%, pero el del último percentil lo hizo un 137\%, y el de los noventa y nueve restantes un $17 \%$. En otras palabras, el $1 \%$ de mayores ingresos se apropió del 55\% del crecimiento ocurrido en dicho período. Como consecuencia de este comportamiento de los ingresos, los indicadores de desigualdad muestran un deterioro. Así, el índice de Gini pasó de 0,44 a 0,57 y la porción de ingreso total apropiada por el $1 \%$ más rico pasó de $14 \%$ a $25 \%$. La retribución al trabajo, en tanto, se redujo de $67 \%$ a $56 \%$ del total (Rodríguez Weber 2014).

Una de las características del crecimiento económico de la década anterior a la Primera Guerra Mundial fue que el mismo se asoció a un fuerte incremento en los precios al consumo que afectó los salarios reales. La tendencia alcista de los precios estaba motiva$\mathrm{da}$-al menos en parte- por la expansión de la masa monetaria real (M2) que entre 1904 y 1907 se incrementó en un 51\%. Los precios al consumo, por su parte, crecieron en esos mismos años un 74\% (Díaz 2010; Matus 2012). La contracara de este crecimiento de los precios fue la caída del salario real de los trabajadores no calificados, que en esos tres años se redujo en un $28 \%$. Si bien entre 1909 y 1912 se produjo cierta mejora, ésta no alcanzó para recuperar lo perdido. Además, los salarios volvieron a caer como consecuencia de la caída del empleo que se produjo a partir de 1913. También la recuperación de 1919-1921 se vio truncada, de modo que no alcanzaron el nivel de 1903 en todo el período (Gráfico 3).

De este modo, la incidencia de la inflación sobre el ingreso de los trabajadores supuso su exclusión de los beneficios asociados al crecimiento económico que se produjo en la década anterior a la Primera Guerra, el que fue canalizado hacia los sectores de la cúspide. Dado que casi el $90 \%$ de la canasta de consumo se componía de productos en que la producción local era muy importante -alimentos, 
vestuario, velas, carbón (Matus 2012, Tablas 16 y 17)-, los cambios en el IPC reflejaban en gran medida variaciones de precios correlacionados con los ingresos de terratenientes, industriales o mineros, categorías que se ubicaban en el 1\% de mayores ingresos.

Si hiciéramos abstracción de los efectos de la política monetaria sobre la inflación, podríamos suponer que la caída del salario real era un resultado de las fuerzas del mercado, pero cuando los trabajadores -recientemente organizados en sindicatos- pugnaron por incrementos nominales que protegieran sus ingresos reales, vieron cómo el Estado ponía la fuerza pública al servicio de sus empleadores. De ese modo, no fue ni el mercado ni las instituciones, sino su articulación en la economía política de la República Oligárquica lo que hizo de la inflación un mecanismo distributivo regresivo.

Gráfico 3: Número total de huelgas y salario real anual de trabajadores urbanos no calificados (Escudos de 1960) entre 1900 y 1925

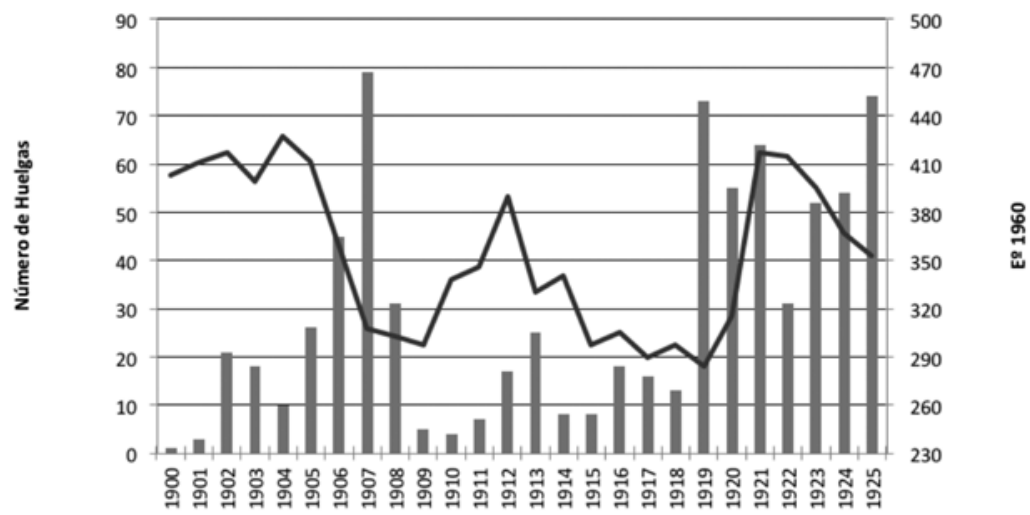

Huelgas $\quad$ Salario de no calificados

Fuente: Huelgas (Pizarro 1988, cuadros 2 y 7); Salarios, estimación propia.

La pérdida de capacidad de compra del salario debida al aumento de los precios, fue el principal detonante de los conflictos obreros durante la primera década del siglo XX (Pizarro 1986). De este modo, el pico inflacionario de 1905-1907 se constituyó en un estímulo para la conformación de organizaciones sindicales (DeS- 
hazo 2007). El Gráfico 3 muestra cómo, en esos años, la caída de los salarios reales fue enfrentada con un incremento en la actividad huelguística. La respuesta del Estado chileno ante las huelgas obreras fue característica de la época y el carácter oligárquico del mismo. Las huelgas provocan desorden y cuestionan a la autoridad establecida; fueron vistas por tanto como una cuestión policial, y sus dirigentes llamados «agitadores», bandidos y delincuentes.

El poder estatal intervino en los conflictos laborales en las dos regiones en que se asentaba el recién nacido movimiento obrero: el norte minero y las ciudades del Valle Central. Sin embargo, lo hizo de forma diferente. En Santiago y Valparaíso la intervención estatal consistió, entre otras cosas, en la infiltración de las organizaciones sindicales mediante la policía y la elaboración por parte de ésta de listas negras con los nombres de dirigentes obreros, las que eran proporcionadas a las empresas. Ocasionalmente, también se utilizaba a la policía o militares como rompehuelgas, aunque en las huelgas pequeñas por lo general no se intervenía (DeShazo 2007, 185-192). También se recurría a la fuerza, especialmente en algunas ocasiones en que las movilizaciones despertaron el pánico entre la elite. Ello ocurrió en octubre de 1903 cuando una multitudinaria manifestación de protesta por la introducción de un impuesto a las importaciones de carne asumió características violentas y fue duramente reprimida, lo que provocó la muerte de un número no determinado de trabajadores (DeShazo 2007, 186). Pero fue en la región minera, que albergaba la piedra angular del sistema económico, donde la violencia constituyó la respuesta estatal normal, lo que resultó en más de una masacre de trabajadores. La más importante fue la ocurrida en la escuela Santa María de Iquique el 21 de diciembre de 1907. Ella ejemplifica tanto la inquietud obrera ante el deterioro del poder de compra de sus ingresos, como la forma en que el Estado y las clases propietarias - nacionales y extranjeras- lo afrontaban. Los trabajadores reclamaban un salario valuado en un tipo de cambio fijo respecto de la libra esterlina en la medida que, señalaban con razón, era en dicha moneda que se valoraba la mercancía que producían. Para entregar su petición, los huelguistas bajaron de la 
Pampa hasta la ciudad de Iquique, donde terminaron instalados en la escuela Santa María. Una vez allí se les ordenó que abandonaran la ciudad, pero se negaron a hacerlo hasta recibir respuesta a sus peticiones. Entonces, por el delito de no obedecer cuando se les mandaba, fueron masacrados por unidades del ejército.

Desde la perspectiva de los actores hegemónicos, lo que estaba en juego no era un incremento de los costos de la producción minera, sino un cuestionamiento a su autoridad y por tanto al poder que de ella emanaba ${ }^{5}$. Ello surge tanto de las declaraciones del intendente de Iquique, Carlos Eastman, como de las del militar encargado de la represión, el General Silva Renand. Para el primero, acceder a las peticiones obreras bajo la presión que suponía su presencia en la ciudad implicaba debilitar el "prestigio moral, el sentimiento de respeto que es la única fuerza del patrón respecto del obrero" (citado por Grez Toso 2007). Para el segundo, la descarga de metralla sobre los obreros se justificaba porque "no era posible esperar más tiempo sin comprometer el respeto y prestigio de las autoridades y la fuerza pública” (citado por Grez Toso 2007). La identificación del Estado con los intereses de la elite fue explicitada, luego de la masacre de Iquique, por el Ministro del Interior de Interior Rafael Sotomayor, quién en comparecencia ante la Cámara de Diputados justificó la acción del Ejército aduciendo que "el fin primordial de un gobierno debe ser el de amparar la propiedad, la vida y el orden social”. En su opinión, si bien "se dice que el patrón se impone al trabajador, [es] el operario es quien se impone al patrón. Las explotaciones de las que se habla existen muchas veces en la fantasía de algunos" (citado por Ortiz Letelier 2005, 155).

La masacre de Iquique supuso el asesinato de un número cercano a las 2.500 personas entre trabajadores y sus familias. Si a ello se agregan las demás masacres producidas no sólo en el norte, sino también en Valparaíso y Santiago, la cifra de obreros muertos entre 1903 y 1907 asciende a unos 3.000 (Pizarro 1986, 20), número similar en magnitud a las bajas chilenas durante la Guerra del Pacífico $^{6}$. A partir de la masacre de Iquique, y en un contexto en que la 
prensa obrera de todo el país llamaba a moderar los reclamos y reducir las acciones de lucha ${ }^{7}$, se produjo una fuerte caída de la agitación sindical. Se inició así un período de reflujo en el movimiento obrero que duraría más de una década (Gráfico 3). Luego de la matanza de Iquique, el poder de compra del salario siguió deteriorándose. Para la década que sigue a la masacre, el salario registra una pérdida promedio de $17 \%$ en comparación con la década anterior (1898-1907), y del 22\% en comparación con el quinquenio anterior al brote inflacionario de 1901-1905 (estimación propia). Mientras tanto, la desigualdad siguió incrementándose, alcanzando su pico más alto hacia 1914, en medio del reflujo del movimiento obrero (Gráficos 2 y 3).

Casi setenta años más tarde, el Estado volvía a poner su fuerza al servicio del incremento de la desigualdad, en este caso como parte de un ajuste estructural que tenía por objeto revertir las mejoras en los ingresos y la distribución producida en los ańos anteriores. De hecho, los elevados niveles que Chile muestra hoy en términos de desigualdad se alcanzaron entonces, en un contexto político altamente represivo, con prohibición y represión de partidos políticos, sindicatos y en general cualquier tipo de oposición, y como resultado de una transformación radical del sistema económico. Como los crímenes cometidos por el régimen dictatorial son ampliamente conocidos, no viene al caso seńalarlos detenidamente aquí ${ }^{8}$, pero sí es importante tenerlos presente como contexto general en que se produjo el incremento de la desigualdad.

Hay que reconocer, sin embargo, que el escenario en el que los Chicago Boys asumieron la conducción de la política económica durante la dictadura de Pinochet era francamente caótico. En los dos últimos ańos del gobierno democrático -1972 y 1973-, el PIBpc cayó un 2,9\% y 7,2\%, el déficit fiscal se descontroló, alcanzando el $24,5 \%$ y $30,5 \%$ del PIB, y los precios se dispararon, incrementándose en un 255\% y 606\% respectivamente (Meller 1998, 131; Díaz et al. 2010). En este marco, la principal preocupación de corto plazo era controlar la inflación y el déficit fiscal. Para lo primero se eliminaron los controles de precios y se aplicó 
una política monetaria contractiva. Se esperaba que, aunque los precios pudieran subir en un primer momento como consecuencia de la liberalización -algo que efectivamente ocurrió-, la contracción monetaria los contendría una vez llegaran al equilibrio. Sin embargo, la inflación se mostró mucho más persistente de lo esperado, manteniendo niveles de tres dígitos hasta 1976. Así, la principal consecuencia de la política monetaria fue una profunda recesión que provocó una caída de 14\% del producto en 1975 (Meller 1998: cuadro 3.3). A ella colaboró además una drástica reducción del gasto público tendiente a eliminar el déficit fiscal. Como era de esperar, la coyuntura recesiva disparó el desempleo y ello, conjugado con la alta inflación y el contexto represivo, afectó los salarios reales. Fue en esta coyuntura que el índice de Gini presenta un cambio de nivel (Gráfico 2 y Tabla 1). Y en todos estos años no se produjo ni una huelga (Tabla 2 ).

En 1978 el PIB per cápita superó el nivel de 1974 y la inflación iba camino a ser controlada. Al año siguiente, el déficit fiscal se transformó en superávit (Díaz et al. 2010) y el salario real superó el nivel de 1973 (Jadresic 1990, Cuadro 1). Se habla entonces de un "milagro chileno». El modelo de crecimiento se caracterizó por un boom de consumo basado en la expansión del crédito. Ambos fueron facilitados por reformas en la esfera financiera y comercial y un rezago cambiario 9 que abarataron las importaciones. Estas crecieron a una tasa del 26\% anual entre 1976 y 1981, y aunque condujeron a un fuerte déficit de la cuenta corriente, el mismo era financiado con creces por un incremento en la entrada de capitales que triplicó la deuda externa en cuatro años. Sin embargo, el desequilibrio externo y el elevado endeudamiento hacía que la situación fuera cada vez más insostenible. En 1981 debieron ser intervenidas las dos principales instituciones bancarias en un contexto de crecientes quiebras de empresas y deudas impagas. Al año siguiente, la economía enfrentó la principal crisis desde 1930. Ese ańo el producto cayó un 15\% y las quiebras se dispararon, amenazando con el hundimiento del conjunto del sistema financiero. 
Tabla 1: Salarios reales, desempleo, inflación y desigualdad (1974-1978)

\begin{tabular}{|c|c|c|c|c|c|c|c|c|}
\hline & $\begin{array}{l}\text { Variación } \\
\text { salario }\end{array}$ & $\begin{array}{r}\text { Variación S } \\
\text { Indus }\end{array}$ & $\begin{array}{l}\text { lario real } \\
\text { ria }\end{array}$ & $\begin{array}{r}\text { Variación d } \\
\text { pú }\end{array}$ & $\begin{array}{l}\text { salario real } \\
\text { co }\end{array}$ & Desempleo & Inflación & Gini \\
\hline Año & Total & Empleados & Obreros & Empleados & Obreros & & & \\
\hline 1974 & $-21 \%$ & $-18 \%$ & $-9 \%$ & $-14 \%$ & $8 \%$ & $9 \%$ & $369 \%$ & 0,453 \\
\hline 1975 & $-5 \%$ & $-17 \%$ & $-14 \%$ & $-16 \%$ & $-7 \%$ & $16 \%$ & $343 \%$ & 0,476 \\
\hline 1976 & $5 \%$ & $8 \%$ & $12 \%$ & $0 \%$ & $-5 \%$ & $19 \%$ & $198 \%$ & 0,530 \\
\hline 1977 & $9 \%$ & $12 \%$ & $16 \%$ & $17 \%$ & $18 \%$ & $18 \%$ & $84 \%$ & 0,529 \\
\hline 1978 & $6 \%$ & $8 \%$ & $8 \%$ & $2 \%$ & $-1 \%$ & $17 \%$ & $37 \%$ & 0,526 \\
\hline
\end{tabular}

Fuentes: salarios, Jadresic (1990, cuadro 2), desempleo e inflación, Meller (1998, cuadro 3.3), Gini, estimación propia a partir de encuesta de Empleo e ingresos para Gran Santiago.

Tabla 2: Represión a organizaciones sindicales

\begin{tabular}{|c|c|c|c|c|}
\hline & Sindicatos & Afiliados & Huelgas & Trabajadores en Huelgas \\
\hline 1970 & 4.581 & 627.664 & 1.853 & 1.860 .662 \\
\hline 1971 & 5.212 & 782.494 & 2.690 & 329.925 \\
\hline 1972 & 6.118 & 855.404 & 3.037 & 440.298 \\
\hline 1973 & 6.692 & 939.319 & 2.230 & 920.737 \\
\hline 1974 & 7.069 & 947.093 & 0 & 0 \\
\hline 1975 & 7.181 & 940.810 & 0 & 0 \\
\hline 1976 & 7.166 & 939.255 & 0 & 0 \\
\hline 1977 & 7.077 & 916.569 & 0 & 0 \\
\hline 1978 & 6.797 & 982.670 & 0 & 0 \\
\hline 1979 & 7.329 & 581.483 & 38 & 10.895 \\
\hline 1980 & 4.597 & 386.910 & 52 & 18.256 \\
\hline 1981 & 3.977 & 395.951 & 82 & 24.504 \\
\hline 1982 & 4.048 & 347.470 & 11 & 1.070 \\
\hline 1983 & 4.401 & 320.903 & 40 & 5.605 \\
\hline 1984 & 4.714 & 343.329 & 39 & 3.685 \\
\hline 1985 & 4.994 & 360.963 & 40 & 4.468 \\
\hline 1986 & 5.391 & 386.987 & 39 & 3.816 \\
\hline 1987 & 5.883 & 422.302 & 124 & 33.642 \\
\hline 1988 & 6.446 & 446.194 & 113 & 32.568 \\
\hline 1989 & 7.118 & 507.616 & 150 & 158.441 \\
\hline 1990 & 8.861 & 606.812 & 321 & 82.438 \\
\hline
\end{tabular}

Fuente: Díaz et al. 2010 
Entonces, y al igual que en la segunda mitad de la década del setenta, las medidas adoptadas para enfrentar la situación tuvieron un claro sesgo regresivo. Se adoptaron políticas recesivas para reducir la demanda agregada y generar los recursos necesarios para pagar la deuda externa. Mientras la política contractiva afectaba en particular al gasto social, el desempleo volvía a dispararse y el salario real a derrumbarse (Tabla 3).

Más aún, la caída del salario real fue de hecho una de las claves adoptadas para superar la crisis, en tanto se esperaba que permitiera reducir la demanda agregada y facilitara la generación del superávit de cuenta corriente necesario para pagar las obligaciones con el exterior. Ello porque un nivel salarial deprimido permitiría reducir las importaciones, mantener contenida la inflación a pesar de la devaluación, y mejorar la competitividad de las empresas chilenas (Meller 1998). Como consecuencia, el índice de Gini tuvo un nuevo repunte en los años finales de la dictadura (Gráficos 1 y 2). Y mientras en los ańos del «milagro», en un contexto de recuperación del salario real, la actividad sindical parecía tener un leve resurgir, a partir de 1982 se produjo un nuevo retroceso (Tabla 2).

Tabla 3: Aspectos distributivos de la crisis de 1982

\begin{tabular}{|r|c|c|c|}
\hline & $\begin{array}{c}\text { Índice de Salario } \\
\text { real }(1982=100)\end{array}$ & $\begin{array}{c}\text { Desempleo } \\
(\%)\end{array}$ & $\begin{array}{c}\text { Indice de ingreso mínimo } \\
\text { liquido (1982=100) }\end{array}$ \\
\hline Año & $(1)$ & $(2)$ & $(3)$ \\
\hline 1982 & 100 & 26,1 & 100 \\
\hline 1983 & 90 & 31,3 & 78 \\
\hline 1984 & 90 & 24,7 & 66 \\
\hline 1985 & 85 & 21,7 & 63 \\
\hline 1986 & 88 & 17,3 & 60 \\
\hline
\end{tabular}

Fuentes: columna 1: Jadresic (1990, cuadro 1), columnas 2 y 3: Meller (1998, cuadros 3.27 y 3.28$)$ 
Pero no sólo las medidas tomadas para afrontar las situaciones críticas tenían un sesgo regresivo. También lo tenía la orientación global de las reformas económicas, lo que explica el apoyo que el empresariado brindaba al gobierno. Así ocurrió, por ejemplo, con la reforma laboral. Los principales mecanismos que median entre la política laboral y la distribución del ingreso son de dos tipos. Por una parte, el Estado puede incidir directamente en el nivel y crecimiento salarial, por ejemplo mediante su política de reajustes o la fijación del salario mínimo. Pero más importante es que al fijar las reglas de relacionamiento entre los actores, la política pública condiciona fuertemente el poder relativo de los mismos, agudizando o atemperando las asimetrías que existen entre empresarios y trabajadores e incidiendo, por esta vía, en la distribución del «ingreso de mercado». La dictadura apeló a ambos mecanismos.

Desde su inicio, el régimen militar se propuso reinstaurar en las empresas la disciplina de los trabajadores y la autoridad de los patrones. Para ello, en un primer momento recurrió a la represión. En 1979, obligada en parte por la presión internacional -y a la vez que mantenía las violaciones a los derechos humanos-, la dictadura impuso el «Plan Laboral». Su consecuencia fue una institucionalización en el plano formal de las relaciones de poder instaladas mediante el terror. Así, el Plan se proponía -y consiguió-, generar un sistema de relaciones laborales que, además de flexibilizar la contratación y el despido de trabajadores, debilitaba a los sindicatos y consagraba el poder de los empresarios ${ }^{10}$. Respecto al salario mínimo, la dictadura lo redujo a un nivel tal que si mantuvo algún efecto en la fijación de los salarios fue colaborar con su caída. Su poder de compra se derrumbó luego del golpe de estado, al punto que para 1975 representaba apenas el 40\% del valor de 1970, último año del gobierno del democratacristiano Frei Montalva. Aunque se recuperó a partir de entonces, el salario mínimo nunca llegó a representar más que el $60 \%$ del de aquel año. Más aún, luego de 1982 volvió a derrumbarse, por lo que en 1989 tenía un nivel apenas superior al de 1975 y equivalente al 42\% del de 1970 (Coloma y Rojas 2000: Cuadro 2). 
En suma, la distribución del ingreso que se deriva de las condiciones del mercado de trabajo depende, además de las condiciones de oferta y demanda, de las instituciones formales e informales que lo regulan, así como de las relaciones de poder entre los agentes. Lo que hizo el Estado de Chile en los dos períodos analizados, fue incidir en estos planos en un sentido favorable a la elite empresarial, poniendo tanto su capacidad de regulación como su poder coercitivo, al servicio de una política de reducción del salario real. La única diferencia es que, si en el primer período ello supuso que el crecimiento del ingreso se concentrara en manos de la elite, en el segundo hizo de los trabajadores las principales víctimas de las crisis. De ese modo, fuera distribuyendo ganancias en un caso, o pérdidas en el otro, el resultado en términos de la desigualdad de ingreso fue el mismo.

\section{FAVORECIENDO LA CONCENTRACIÓN DE LA PROPIEDAD Y EL LUCRO} Luego de 1880, el Estado chileno tomó control efectivo sobre el territorio de la Araucanía, ubicado al sur de la zona de poblamiento tradicional. En los veinte años siguientes, la región conoció un crecimiento poblacional acelerado. Entre 1875 y 1907, los habitantes de La Frontera y Los Lagos pasaron de representar el 5\% al 17\% de la población total de Chile. El crecimiento de la población era consecuencia de la migración de miles de colonos que se acercaron hasta allí en busca de tierras durante el período de indefinición de los derechos de propiedad que siguió a la conquista de la Araucanía.

La colonización del sur fue el resultado de la combinación de tres vertientes: dos estrategias públicas y un proceso espontáneo. En cuanto a las primeras, el Estado se debatió entre dos alternativas. Por un lado se buscaba la colonización por parte de extranjeros y chilenos que trabajasen su propia tierra. En este marco se entregaron tierras a oficiales y tropa del ejército desmovilizado, y «mercedes» a los indígenas. La segunda alternativa era defendida por quienes sostenían que el Estado debía abstenerse de intervenir, dejando el proceso de colonización librado a lo que resultara de la libre compra de tierras. Pero también se instalaron numerosos "colonos espontáneos», animados por la promesa de tierras. Esta situación, en que la incertidumbre 
sobre los derechos de propiedad generaba una ventana de oportunidad para los trabajadores rurales pobres del Valle Central dio paso, a principios del siglo XX y gracias a la acción estatal, a la concentración de la propiedad y la consolidación del latifundio.

Este proceso fue consecuencia de las políticas de subastas y colonización adoptadas por el Estado, así como de la corrupción que caracterizó a su ejecución. Como la legislación impedía la subasta de predios mayores a 400 hectáreas y prohibía que una misma persona comprara más de 2.000 , se recurría a los servicios de intermediarios o especuladores conocidos como "palos blancos», los que remataban a favor de terceros (Bengoa 1990). También se compraba tierras a ocupantes pobres y sin títulos o a extranjeros que, aunque habían sido traídos por las empresas de colonización agrícola, eran en realidad artesanos de origen urbano (Johnson 1978; Solberg 1969).

Pero más significativo fue el rol de la política de colonización. Se trató probablemente de la principal herramienta de consolidación de derechos de propiedad en el sur, y tuvo un claro signo regresivo. En primer lugar, porque desde su diseño discriminaba a los pobres del país, especialmente si pertenecían a los pueblos originarios. La elite gobernante veía en los «rotos», y mucho más en los indígenas, a un conjunto de vagos y borrachos, personas incapaces de llevar adelante un proceso de modernización en la frontera, y recurría a «argumentos» racistas para fundamentar la necesidad de importar trabajadores europeos (Solberg 1969). En cuanto a la población originaria, se les brindaba apenas lo necesario: entre 1884 y 1929 se entregaron títulos de merced por 504.778 hectáreas a 90.401 personas, es decir algo más de 5 ha a cada uno (Almonacid 2009, cuadro 1). En segundo lugar, porque aunque teóricamente diseñada para poblar la región del sur con colonos europeos, su principal resultado fue entregar enormes extensiones de tierras a quienes se suponía debían traerlos.

Las dimensiones de las concesiones fueron enormes. Durante el primer gobierno del siglo XX, dirigido por el Presidente Riesco entre 1901 y 1905, se otorgaron 46 concesiones por un total de 4 millones y medio de hectáreas (Bengoa 1990, 167 nota 23), es decir una cifra nueve veces mayor que la devuelta a la población originaria a 
lo largo de treinta años. A cambio de promover la inmigración, los concesionarios se quedaban con enormes extensiones de tierra. La Empresa Colonizadora Budi, por ejemplo, recibía 150 hectáreas por familia que instalara, de los cuales entregaba $75 \mathrm{Ha}$ a los colonos. Algo similar ocurrió con el empresario de origen italiano Jorge Ricci, quien en 1903 recibió 27.000 hectáreas, de las cuales podía retener 10.400. Pero era habitual que incluso las tierras previstas para los colonos terminaran formando latifundios. En 1905, por ejemplo, el gobierno entregó a Luis Silva Rivas 200.000 hectáreas de tierras ocupadas por comunidades indígenas y colonos nacionales espontáneos que se habían instalado allí luego de la ocupación de la Araucanía. El gobierno habilitó al concesionario a radicar a los «ocupantes» en otro lugar, obligando a los indígenas a abandonar sus tierras ancestrales y a los colonos los campos que "habían abierto, limpiado y sembrado" (Bengoa 1990, 171). La sociedad concesionaria no cumplió con la instalación de colonos. Cuando se vio obligada a salvar las apariencias, contrató un grupo de inmigrantes europeos, pero ninguno era agricultor, sino que tenían oficios urbanos. Además, se les obligó a hipotecar sus haciendas a favor de la Sociedad Silva Rivas (Bengoa 1990, 172). Otro caso es la ya mencionada Empresa Colonizadora Budi. Aunque la empresa se había comprometido a instalar trescientas familias, finalmente fueron cien, e incluso éstas tampoco se radicaron, de modo que en 1939 no había ninguna (Solberg 1968, 125).

Las empresas no sólo no eran sancionadas por no cumplir sus compromisos sino que de hecho se beneficiaban al quedarse con las tierras que debían destinar a los colonos. Ese es el caso de 8 empresas analizadas por Almonacid $(2009,15)$. A principios de siglo éstas recibieron un total de casi 214.000 hectáreas para fincar colonos en Cautín, Mallco y Validvia. Instalaron 363 familias que ocuparon 28.165 hectáreas en las dos últimas de las provincias mencionadas. Las otras 185.835 quedaron en manos de las empresas «colonizadoras». De forma similar, de las 12.200 familias que se suponía ocuparían las 46 concesiones hechas durante el gobierno del presidente Riesco y a las que hicimos referencia más arriba, sólo llegaron 47 (Solberg 1969, 126). 
Además, a la hora de regularizar la situación se adoptaba un doble estándar en función de quién estuviera involucrado. En los casos de aquellos "propietarios» que habían subastado o "comprado», habitualmente en condiciones irregulares, la política seguida fue regularizar la posesión de hecho (Almonacid 2009). Pero la situación era diferente para los pobres. En estos casos -como el de la Sociedad Silva Rivas-, se recurría a la fuerza pública para expulsar a los ocupantes e imponer a los nuevos dueños. De este modo, la expansión del latifundio en la Araucanía fue el resultado de la inercia institucional representada por la longevidad del sistema hacendal, junto con la acción política de un Estado controlado en gran medida por terratenientes. Para 1930, la región que había brindado sustento a los mapuches, y que había sido tierra de oportunidad para miles de colones pobres provenientes del Valle Central, albergaba los más grandes latifundios de Chile (estimación propia).

Algo similar ocurriría siete décadas más tarde, pero en este caso los bienes públicos privatizados en oscuras condiciones fueron las empresas públicas, sector que había crecido en forma importante durante las décadas de Industrialización Dirigida por el Estado. La primera oleada de privatizaciones, ocurrida en los años setenta, permitió que algunos grupos empresariales se adueńaran de una enorme cantidad de empresas que habían pasado a control del Estado. Se trataba de compras realizadas muchas veces a crédito, por lo que estas empresas comenzaron con un alto nivel de endeudamiento, lo que condujo a su quiebra durante la crisis de 1982. Entonces debieron ser nuevamente nacionalizadas, dando lugar a lo que se denominaba el área "rara" de la economía o, lo que algunos llamaron con sorna, la "vía Chicago al socialismo" (Stallings 2001, 36). La segunda -y más importante- oleada de privatizaciones se produjo durante los últimos años de la dictadura. Esta abarcó no sólo a las empresas nacionalizadas como consecuencia de la crisis de 1982, sino a empresas de servicios públicos tradicionalmente en manos del Estado que no habían sido privatizadas hasta entonces por la oposición de sectores de las Fuerzas Armadas que las consideraban estratégicas para la seguridad nacional. El nuevo proceso privatizador recogió alguna de las enseñanzas del pasado -por ejemplo no se permitió la compra a crédito-, pero fue -por decir lo menos- muy poco transparente, dando lugar a ventas a pre- 
cios muy inferiores a los de mercado, o al aprovechamiento por parte de algunos funcionarios de información privilegiada que les permitió convertirse en dueños de las empresas privatizadas (Meller 1998, 270-271; Monckeberg 2001; Gárate Chateau 2012, 308-316).

La otra fuente de beneficios que obtuvo la elite de las reformas estructurales efectuadas durante la dictadura fue la privatización de servicios vinculados a las funciones secundarias del Estado -salud, pensiones, educación-, la que abrió al mercado, y por tanto al lucro, toda una nueva área de la vida social. A partir de entonces las ISAPRES $^{11}$, las $\mathrm{AFP}^{12}$, o las Universidades Privadas -teóricamente sin fines de lucro-, se han convertido en parte de la vida cotidiana de los chilenos y en una fuente de ganancias fantásticas para sus dueños y de salarios extraordinariamente altos para su plana gerencial. Se trata además de mercados altamente concentrados, lo que posibilita la obtención de rentas monopólicas a costa de los consumidores, quienes resultan perjudicados por las enormes asimetrías de información que existen entre ellos y las empresas que les venden los servicios. De este modo, el "capitalismo a la chilena"13 originado durante el período dictatorial, y por tanto en un contexto institucional muy peculiar, no dio lugar precisamente a los mercados atomizados y la libre competencia que anunciaban los Chicago Boys, sino a una estructura de merados altamente concentrada, donde un pequeño grupo de empresas emparentadas entre sí controlan la producción de transables y no transables, entre ellos algunos servicios tradicionalmente provistos por el Estado (Solimano 2012).

En suma, en los dos períodos analizados, el Estado tomó una serie de decisiones de política que propiciaron la concentración de la propiedad y la expansión del lucro. La transferencia de la propiedad de bienes públicos, fueran tierras o empresas, a -unas pocas- manos privadas y en un contexto altamente opaco y signado por la corrupción, fue un mecanismo común a ambos episodios de incremento de la desigualdad. Durante la dictadura, además, se privatizó también la provisión de servicios públicos antes considerados derechos de los ciudadanos, ampliando enormemente -como consecuencia- el área de la economía en que los miembros de la elite podían obtener 
lucro. $\mathrm{Y}$ al igual que en el caso de las ex empresas públicas, también aquí el proceso estuvo signado por la corrupción y la concentración de los mercados.

\section{Conclusiones}

Hasta hace pocos ańos, la mayor parte de la literatura sobre la evolución de la desigualad de ingresos -tanto en América Latina como a nivel global- ha supuesto que los cambios en la oferta y demanda de factores resultan suficientes para explicar la distribución del ingreso de mercado. Esta literatura suele limitar la incidencia del Estado a la redistribución por la vía de impuestos, transferencias y gastos. Sin embargo, el Estado contribuye también a moldear la distribución primaria tanto por su incidencia en las instituciones que regulan los mercados de factores -salario mínimo, derechos de propiedad, régimen de negociación colectiva, regulación de la actividad sindical, etc.- como por su participación directa en el conflicto distributivo entre los actores económico-sociales. Dicha incidencia interactúa con los factores de mercado en un sentido que puede reforzar, aminorar, o revertir las tendencias distributivas que, en ausencia de factores institucionales, se derivarían de la evolución de la oferta y la demanda.

El artículo hace uso de estadísticas históricas de ingreso y desigualdad para realizar un análisis comparativo de los mecanismos de economía política que contribuyeron a generar un fuerte deterioro de la distribución del ingreso en dos momentos de la historia de Chile. El vínculo privilegiado entre las elites y quienes dirigían el Estado, -originado en el período colonial y reforzado luego de la Independencia- fue en ambos casos un factor determinante del resultado distributivo observado. Los principales mecanismos actuantes en ambos períodos refieren, por un lado, a la represión de movimientos políticos y sindicales que desarmaron a los trabajadores en contextos inflacionarios, lo que dio lugar a una evolución de los precios relativos ampliamente perjudicial para los asalariados. A ello se agrega, en el segundo período, la imposición de un régimen de relaciones laborales que tenía por objetivo revertir la evolución 
favorable a los trabajadores que en ese plano se había observado en las décadas anteriores. La privatización de bienes y servicios públicos, realizada en un contexto signado por la corrupción -la que era facilitada en ambos casos por la ausencia de los controles que caracterizan a la democracia- constituye el segundo mecanismo por el cual la acción política favoreció la concentración de la propiedad, la expansión del lucro, y el deterioro de la distribución de los ingresos «de mercado». En el período reciente, este proceso ha dado lugar a un estilo particular de capitalismo, caracterizado por la mercantilización de servicios que otrora fueran considerados derechos, los que son provistos por empresas que actúan en mercados oligopólicos -lo que les permite obtener importantes rentas a costa de los consumidores-.

De cara a una futura agenda de investigación, el artículo muestra la potencialidad de dos líneas de acción. En primer lugar, la realización de un análisis comparado entre distintos períodos históricos, algo posibilitado -a su vez- por la elaboración de estadísticas históricas de desigualdad. En nuestro caso, ello ha permitido identificar la acción de factores de larga duración, como la tendencia a privatizar y concentrar bienes y servicios públicos, o a utilizar la capacidad coercitiva del Estado para propiciar la caída del salario real, tanto en contextos de crecimiento como de crisis, dirigiendo así una parte creciente de los ingresos "de mercado" a manos de la elite. En segundo lugar, del trabajo surge la pertinencia de considerar los mecanismos institucionales que inciden en los procesos distributivos no sólo cuando se analiza la desigualdad luego de impuestos y transferencias, sino también para comprender las características asumidas por la distribución de los ingresos de mercado.

De lo que se trata, en suma, es de construir una agenda de investigación sobre la economía política de la desigualdad en América Latina; la que, centrada en la interacción de factores institucionales y de mercado, analice las tendencias asumidas por la distribución del ingreso a lo largo del tiempo como parte del proceso histórico general y -por tanto- como fenómeno multicausal. 
Bibliografía

ACEMOGLU, Daron, Simon JOHNSON and James ROBINSON (2005) "Institutions as a fundamental cause of long-run growth." En Handbook of economic growth. Volume 1, editado por Philippe AGHION y Steven N. DURLAUF, 385-472. Amsterdam: Elsevier 1.

ACEMOGLU, Daron and James. A. Robinson (2012) "Why $\mathrm{Na}$ tions Fail: The Origins of Power, Prosperity, and Poverty”. New York: Crown Publishing Group.

ALMONACID, Fabian (2009) "El problema de la propiedad de la tierra en el sur de Chile (1850-1930).” Historia (Santiago) 42 (1): 5-56. ATKINSON, Anthony (2015) "Inequality: What Can Be Done?" Cambridge: Harvard University Press, 2015.

ATKINSON, Anthony (1997) "Bringing income distribution in from the cold." The Economic Journal 107 (441): 297-321.

ATKINSON, Antonhy y François BOURGUIGNON (2007) "Introduction." En Handbook of Income Distribution, editado por Anthony Atkinson y François Bourguignon,1-58. Amsterdam: Elvesier.

BENGOA, José (1990) Haciendas y campesinos. Historia social de la agricultura chilena. Santiago de Chile, Ediciones Sur.

BÉRTOLA, Luis (2005) "A 50 años de la curva de Kuznets: Crecimiento económico y distribución del ingreso en Uruguay y otras economías de nuevo asentamiento desde 1870." IHE Investigaciones de Historia Económica 1(3): 135-176.

BÉRTOLA, Luis (2011) "Institutions and the Historical Roots of Latin American Divergence." En The Oxford Handbook of Latin American Economics, editado por Jaime ROS y José Antonio OCAMPO, 26-49. Oxford, Oxford University Press.

BÉRTOLA, Luis, Cecilia CASTELNOVO, Javier RODRÍGUEZ and Henry WILLEBALD (2010) "Between the colonial heritage and the first globalization boom: on income inequality in the Southern Cone." Revista de Historia EconómicalJournal of Iberian and Latin American Economic History (Second Series) 28 (02): 307-341. 
BEYER, Harald, Patricio ROJAS and Rodrigo VERGARA (1999) "Trade liberalization and wage inequality." Journal of Development Economics 59 (1): 103-123.

COLOMA, Fernando y Patricio ROJAS (2000) "Evolución del mercado laboral en Chile: Reformas y resultados." En La transformación económica en Chile, editado por Felipe B. LARRAÍN, Rodrigo VERGARA, y Rodrigo ACUÑA, 492-540. Santiago de Chile: Centro de Estudios Públicos.

DESHAZO, Peter (2007) Trabajadores Urbanos Y Sindicatos en Chile, 1902-1927. Santiago de Chile: Centro de Investigaciones Diego Barros Arana.

DÍAZ, José, LÜDERS, Rolf y WAGNER, Gert (2010) "La República en Cifras. Santiago de Chile”, EH Clio Lab-Iniciativa Cientifica Milenio. GÁRATE Chateau, Manuel (2012) La revolución capitalista de Chile. Santiago de Chile: Ediciones Universidad Alberto Hurtado. GOLDIN, Claudia D. and Lawrence KATZ (2009) The Race between Education and Technology. Cambridge, MA: Harvard University Press.

GREZ TOSO, Sergio (2001) "La guerra preventiva: Escuela Santa Maria de Iquique las razones del poder." Mapocho; Revista de Humanidades y Ciencias Sociales (50): 271-280.

JADRESIC, Esteban (1990) "Salarios en el largo plazo: Chile 19601989." Colección estudios CIEPLAN (29): 9-34.

JOHNSON, Ann Louise Hagerman (1978) "International migration in Chile to 1929: its relationship to the labor market, agricultural growth, and urbanization”. PhD., University of California, Davis.

KAHHAT, Jaime (2010) "Labor Earnings Inequality: The Demand for and Supply of Skills." En Declining Inequality in Latin America: A Decade of Progress, editado por Luis Felipe López-Calva y Nora Lustig, 25-38. Washington D.C.: Brookings Institution Press.

LINDERT, Peter H. and Jeffrey WILLIAMSON (1982) "Revising England's social tables 1688-1812." Explorations in Economic History 19 (4): 385-408.

LINDERT, Peter H. and Jeffrey Williamson (2014) "American Colonial Incomes, 1650-1774." The Economic History Review (551): n/a. LONDOÑO, José Luis (1995) Distribución del ingreso y desarrollo 
económico: Colombia en el siglo XX. Bogotá: TM Editores.

MATUS, Mario (2012) Crecimiento sin desarrollo: precios y salarios reales durante el Ciclo Salitrero en Chile (1880-1930). Santiago de Chile: Editorial Universitaria.

MELLER, Patricio (1996) Un Siglo de economía politica chilena (1890-1990). Santiago de Chile: Editorial Andrés Bello.

MILANOVIC, Branko (2010) "Level of income and income distribution in mid-18th century France, according to Francois Quesnay." World Bank Policy Research Working Paper (10545). MÖNCKEBERG, María Olivia (2001) El saqueo de los grupos económicos al Estado chileno. Santiago de Chile: Ediciones B.

ORTIZ LETELIER, Fernando (2005) El movimiento obrero en Chile, 1891-1919. Santiago de Chile: LOM Ediciones.

PIKETTY, Thomas (2014) Capital in the Twenty First Century. Cambridge MA: Harvard University Press.

PIKETTY, Thomas (2015) "Putting Distribution Back at the Center of Economics: Reflections on "Capital in the Twenty-First Century"." The Journal of Economic Perspectives: 29 (1): 67-88.

PIZARRO, Crisóstomo (1986) La huelga obrera en Chile: 18901970. Santiago de Chile: Ediciónes Sur.

REINSCH, Paul (1909) "Parliamentary Government in Chile." The American Political Science Review 3 (4): 507-538.

ROBINSON, James (2003) "Where Does Inequality Come From?: Ideas and Implications for Latin America". En Globalisation, Poverty and Inequality, editado por Richard KOHL, 71-75. Paris: Development Centre of the Organisation for Economic Co-operation and Development.

RODRÍGUEZ WEBER, Javier (2014) "La Economía Política de la Desigualdad de Ingreso en Chile, 1850-2009” Tesis de Doctorado en Historia Económica, Programa de Historia Económica y Social, Facultad de Ciencias Sociales, Universidad de la República, Uruguay. ROBINSON, James (2010) "The political economy of redistributive policies." En Declining Inequality in Latin America: A Decade of Progress, editado por Luis Felipe LÓPEZ-CALVA y Nora LUSTIG, 39-71. Washington D.C.: Brookings Institution Press. 
SOLBERG, Carl (1969) "A discriminatory frontier land policy: Chile, 1870-1914." The Americas 26 (2): 115-133.

SOLIMANO, Andrés (2012) Capitalismo a la Chilena y la prosperidad de las élites. Santiago de Chile: Catalonia.

STALLINGS, Barbara (2001) "Las reformas estructurales y el desempeño socioeconómico." En Reformas, crecimiento y politicas sociales en Chile desde 1973, editado por Ricardo FFRENCHDAVIS, Barbara STALLINGS, 23-60. Santiago de Chile: CEPAL/LOM Ediciones, 2001-p. 23-60.

TINBERGEN, Jan (1975) Income distribution: analysis and policies. Amsterdam: North-Holland Pub. Co.

VIAL CORREA, Gonzalo (2010) Chile, cinco siglos de historia: desde los primeros pobladores prehispánicos, hasta el año 2006. Santiago de Chile: Zig-Zag.

\section{Notas de Página}

${ }^{*}$ El presente artículo se basa en los capítulos 2, 7 y 9 de mi tesis doctoral, orientada por Luis Bértola, a quién estoy especialmente agradecido. Deseo asimismo agradecer a: Alfonso Herranz, Branko Milanovic, Brian Loveman, Carolina Román, César Yáñez, Gabriel Oddone, Henry Willebald, José Díaz, José Martínez-Carrión, Jorge Álvarez, Marc Badía, María Camou, Mario Matus, Natalia Pérez Barreda, Paola Azar, Peter Lindert, Reto Bertoni, Rosemary Thorp, Sabrina Siniscalchi, Sebastián Fleitas, Silvana Maubrigades, Tarcísio Botelho, Verónica Amarante, Vicente Neira, y Xavier Taffunel.

${ }^{1}$ Una conclusión que, por otra parte, se ve reforzada por las dificultades que presentan las encuestas para captar ingresos provenientes de otras fuentes.

${ }^{2}$ Para un análisis más profundo de lo ocurrido en este período dónde se incluyen factores de mercado, ver Rodríguez Weber (2014). Para un ejemplo de literatura neoclásica que desdeña el rol de los factores institucionales en el incremento de la desigualdad en Chile luego de 1973 ver Beyer et al. (1999).

${ }^{3}$ Otros ejemplos de este tipo de abordaje son Bértola (2005) y Lindert and Williamson (2014). 
${ }^{4}$ Una descripción detallada de los procedimientos seguidos y las fuentes utilizadas puede encontrarse en Rodríguez Weber (2014).

${ }^{5}$ El Estado se había ofrecido a cubrir la mitad del costo que supondría acceder a la petición de los obreros, lo que -aunado al elevado precio internacional del salitre- hacía que el eventual sacrificio de utilidades fuera irrisorio.

${ }^{6}$ Cifra publicada por el sitio web http://necrometrics.com/ wars 19c.htm\#Pacific consultado en mayo de 2013.

${ }^{7}$ Dos ejemplos. En enero de 1908 el periódico obrerista La Reforma recomendaba a las sociedades de resistencia que no se hicieran reuniones ni se formaran "grupos explosivos" (DeShazo 2007, 178). De forma similar, los adherentes de la Mancomunal de Tocopilla se rehusaron en plebiscito a participar de una huelga en repudio a la masacre que convocaba el Congreso Social Obrero, y señalaban que no era el "momento oportuno para introducir un movimiento huelguístico" (citado por Ortiz Letelier 2005, 153). Hacia mayo, el periódico La Reforma señalaba que, presionados por sus patrones, los trabajadores abandonaban los sindicatos (DeShazo 2007, 178).

${ }^{8}$ Quién desee hacerlo puede consultar el Informe Rettig, disponible en http://www.ddhh.gov.cl/ddhh_rettig.html.

${ }^{9}$ Entre junio de 1979 y el mismo mes de 1982, el tipo de cambio real se deterioró en un 30\% (Meller 1998, 202).

${ }^{10}$ Coloma y Rojas (2000) describen la reforma laboral del período, señalando explícitamente que la misma se proponía reducir el poder negociador de los trabajadores, algo que en su opinión era deseable.

${ }^{11}$ Empresas que otorgan servicios de salud.

${ }^{12}$ Administradoras de fondos previsionales.

${ }^{13}$ Título del libro de Andrés Solimano (2012).

Fecha de Recepción del Artículo: 9 de marzo de 2016 Fecha de Aprobación: 6 de mayo de 2016 